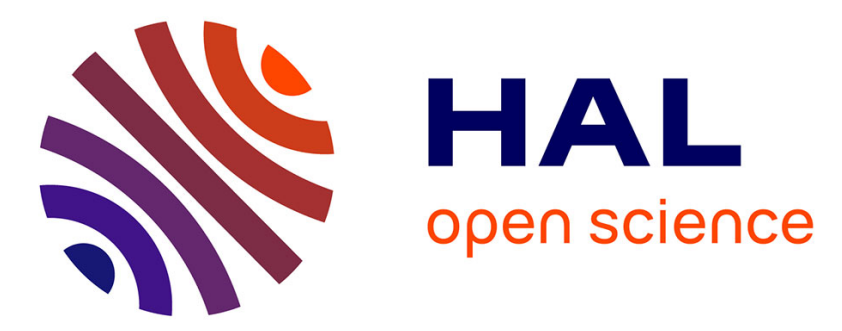

\title{
Structures multicouches gravées pour le rayonnement X-UV
}

\author{
R. Barchewitz, J.-M. André
}

\section{To cite this version:}

R. Barchewitz, J.-M. André. Structures multicouches gravées pour le rayonnement X-UV. Journal de Physique IV Proceedings, 1996, 06 (C4), pp.C4-763-C4-772. 10.1051/.jp4:1996474 . jpa-00254357

\section{HAL Id: jpa-00254357 https://hal.science/jpa-00254357}

Submitted on 1 Jan 1996

HAL is a multi-disciplinary open access archive for the deposit and dissemination of scientific research documents, whether they are published or not. The documents may come from teaching and research institutions in France or abroad, or from public or private research centers.
L'archive ouverte pluridisciplinaire HAL, est destinée au dépôt et à la diffusion de documents scientifiques de niveau recherche, publiés ou non, émanant des établissements d'enseignement et de recherche français ou étrangers, des laboratoires publics ou privés. 


\title{
Structures multicouches gravées pour le rayonnement X-UV
}

\author{
R. Barchewitz et J.-M. André \\ Laboratoire de Chimie Physique, Groupe Optique X, Université Pierre et Marie Curie, URA 176 du \\ CNRS, 11 rue Pierre et Marie Curie, 75231 Paris cedex 05, France
}

\begin{abstract}
Résumé: L'analyse spectrale et l'imagerie du rayonnement X-UV ont depuis environ deux décennies considérablement bénéficié du développement des miroirs interférentiels multicouches à période nanométrique. La possibilité de graver ces structures multicouches à l'aide de techniques de microfabrication (lithographie électronique et $X$, gravure par plasma...) a ouvert plus récemment de nouvelles perspectives dans le domaine de l'optique X-UV. Nous en présentons ici quelques exemples: les réseaux de diffraction lamellaires, les monochromateurs à bande passante étroite, les polychromateurs et enfin les lentilles dites de Bragg-Fresnel.
\end{abstract}

\begin{abstract}
In the last two decades, the development of nanometric period multilayer interferential mirrors has largely contributed to the progress of X-UV spectral analysis and imagery. The ability to pattern the multilayer structures by means of microfabrication technologies (electronic and $x$-ray lithography, plasma etching ..) has, more recently, opened up new possibilities in the area of X-UV optics. Some examples are presented in this paper: the lamellar diffraction gratings, the monochromators with narrow bandwidth, the polychromators and, finally, the so-called Bragg-Fresnel lenses.
\end{abstract}

\section{INTRODUCTION}

Les structures multicouches constituées d'un empilement périodique alterné de deux matériaux offrant un large écart d'indice optique permettent de diffracter efficacement le rayonnement $\mathrm{X}$ UV, en particulier le rayonnement de longueur d'onde supérieure à environ $2 \mathrm{~nm}$ qu'il n'est pas possible de diffracter au moyen de cristaux naturels. Ces miroirs interférentiels multicouches (MIMs) qui obéissent à la loi de Bragg sont maintenant largement utilisés pour l'analyse spectrale, en particulier pour les émissions caractéristiques des éléments légers dans les spectromètres de fluorescence et les microsondes. Cependant pour cet usage, les MIMs présentent un certain nombre d'inconvénients: une bande passante spectrale relativement large dégradant la résolution, une zone de réflexion quasi-totale assez étendue donnant lieu à un fond continu gênant, l'existence de réflexions de Bragg à de multiples ordres occasionnant des problèmes de recouvrement de pics. Nous montrons par la suite que les structures multicouches gravées pourraient pallier certains de ces problèmes. En fait la possibilité de graver ces MMMs suivant un motif périodique ou non permet de réaliser des dispositifs originaux pour l'optique $\mathrm{X}-\mathrm{UV}$. Ainsi la gravure périodique fournit des réseaux de diffraction qui dans certaines conditions présentent des propriétés spécifiques qui peuvent être mises à profit pour concevoir de nouveaux outils. Parmi ceux-ci nous présenterons ce que nous avons appelés le "polychromateur". La gravure non périodique a été utilisée dans la fabrication de lentilles dites de Bragg-Fresnel. Celles-ci permettent la focalisation du rayonnement selon des dimensions micrométriques et pourraient permettre la réalisation d'imagerie.

\section{TECHNIQUES DE FABRICATION}

Les structures multicouches sont fabriquées suivant plusieurs techniques, la plus usuelle compte tenu des matériaux employés étant la pulvérisation et en particulier la pulvérisation magnétiquement assistée. Rappelons que le choix des matériaux est déterminé par le domaine 
spectral et par la faisibilité d'un empilement stable présentant des interfaces assez lisses. Les couples de matériaux W/C, W/Si, Mo/C, Mo/Si sont souvent employés.

La réalisation d'une structure multicouche gravée comprend deux phases principales : l'écriture du motif et la gravure proprement dite. La fabrication suit en fait un processus à plusieurs étapes [1]. Actuellement la maîtrise de l'ensemble du processus reste encore délicate et n'est le propre que d'un nombre très limité de laboratoires. En France, le Laboratoire de Microstructures et Microélectronique du CNRS à Bagneux joue un rôle majeur dans le développement de ces techniques. L'écriture consiste à définir le motif désiré dans la résine déposée préalablement sur la surface de la structure multicouche. Trois techniques ont été jusqu'à présent exploitées: la holographie UV qui permet d'obtenir sur de larges surfaces des réseaux périodiques de faible période (submicrométrique) mais dont le profil est mal maîtrisé, la lithographie électronique qui permet de réaliser des motifs périodiques ou non mais qui reste limitée à de petites surfaces, et la lithographie $X$, en cours de développement, qui devrait autoriser la réalisation sur de larges surfaces de motifs divers après fabrication préalable d'un masque par lithographie électronique; la lithographie $X$ offre l'avantage d'offrir une courte durée de réalisation mais nécessite l'utilisation d'une source intense de rayonnement $X$ (synchrotron ou source plasma). Le transfert du motif dans la structure multicouche est réalisé généralement par une gravure sèche au moyen d'un plasma (Reactive Ion Etching) dont les conditions sont appropriées aux paramètres du motif et à la nature des matériaux.

\section{MIROIRS INTERFERENTIELS MULTICOUCHES}

Les miroirs interférentiels multicouches (structures non gravées) utilisés dans le domaine $X-U V$ miroirs diffractent d'une manière analogue au cristaux naturels et suivent donc la loi de Bragg (cf. Fig.1)

$$
2 \mathrm{~d} \sin \theta_{0}=\mathrm{m} \lambda
$$

où $\lambda$ est la longueur d'onde, $\theta_{0}$ l'angle d'attaque, $d$ la période de l'empilement et $\mathrm{m}$ un entier positif (ordre de réflexion). En fait cette loi s'applique si $\lambda$ et $\theta_{0}$ sont respectivement la longueur d'onde et l'angle dans le milieu optique moyen constitué par les matériaux constituant la multicouche et caractérisé par un indice optique moyen. Si $\lambda$ et $\theta_{0}$ sont estimés dans le milieu incident (le vide généralement), il est nécessaire dans le domaine X-UV d'effectuer une correction d'indice. L'aspect dynamique de la diffraction est très bien décrit par les lois de l'électromagnétisme dans les milieux continus. On peut ainsi calculer la réflectivité, la largeur de bande à l'aide des modèles développés pour traiter l'optique des films minces dans le domaine des grandes longueurs d'onde (UV, visible, IR) [2]. Les principales erreurs sur les valeurs théoriques proviennent généralement de la méconnaissance des valeurs des indices optiques liée à l'incertitude sur la densité des matériaux et à l'incertitude sur les facteurs atomiques de diffusion. Le principe, les méthodes de fabrication, le calcul des performances ainsi que les principales applications des MIMs sont exposés dans la référence [3].

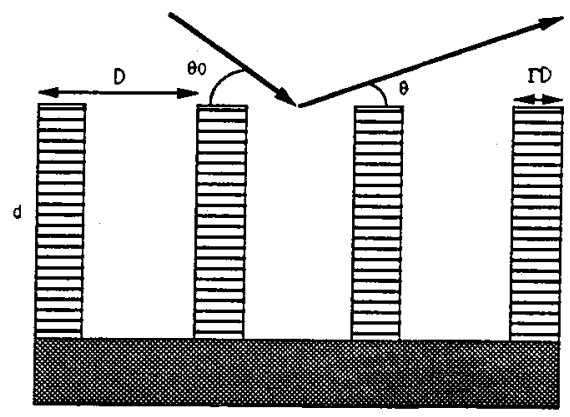

Figure 1: Schéma d'un réseau muiticouche lamellaire Lamellar multilayer grating scheme 


\section{STRUCTURES PERIODIQUES}

\subsection{Diffraction par des réseaux lamellaires multicouches}

Le schéma d'un réseau lamellaire multıcouche est présenté sur la figure 1. Cette structure constitue un système doublement périodique (une périodicité en profondeur correspondant à l'empilement multicouche et une périodicité latérale correspondant à la gravure) susceptible de

\section{Expérience}

\section{Theorie}

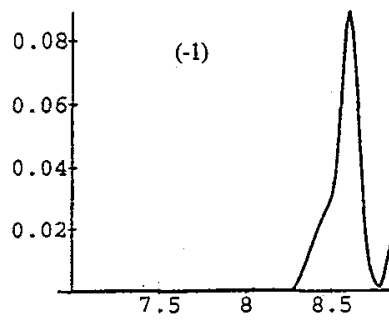

a)
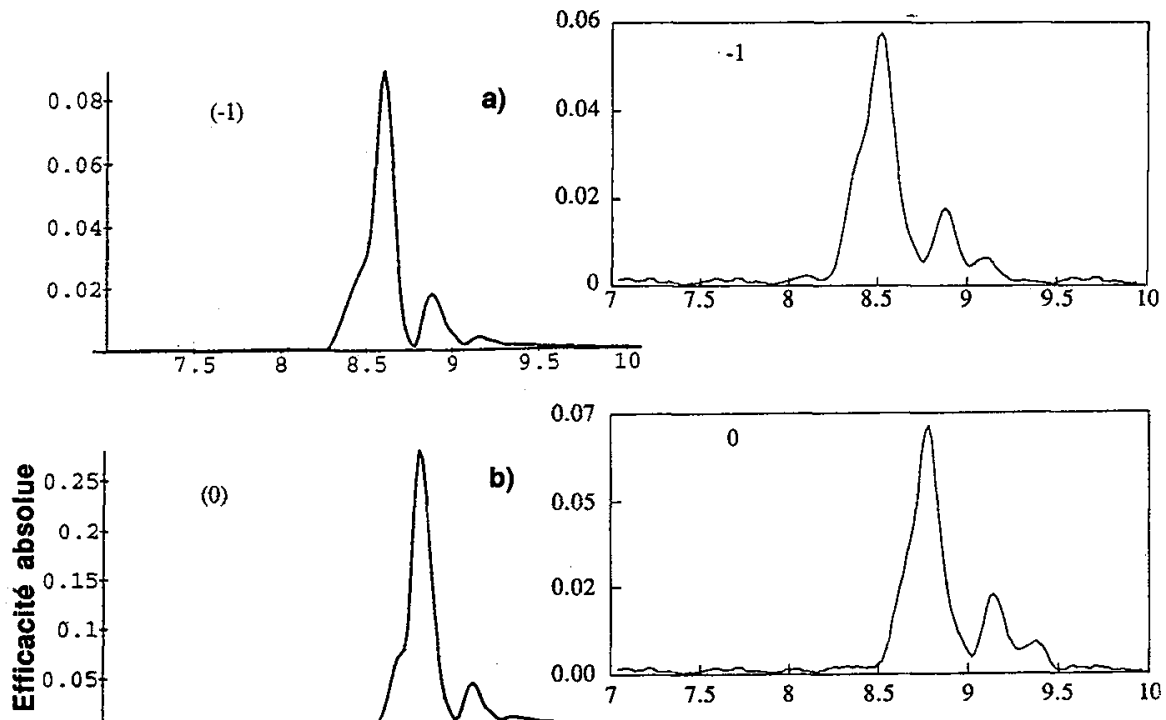

(0)

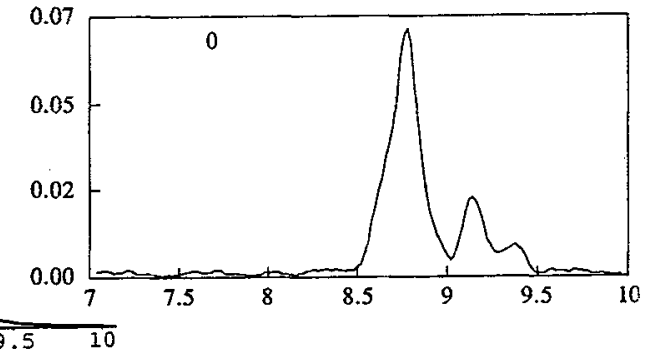

$\begin{array}{llllll}7.5 & 8 & 8.5 & 9 & 9.5 & 10\end{array}$

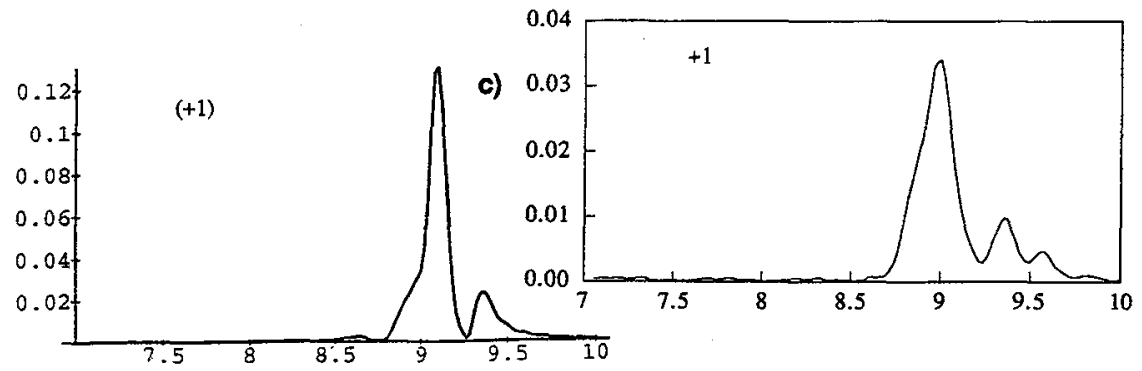

Angle d'attaque $\theta_{0}(\mathrm{deg})$

Angle d'attaque $\theta_{0}$ (deg)

Figure 2: Variations expérimentale et théorique avec l'angle d'attaque des efficacités des ordres -1 (a), 0 (b), +1 (c) d'un réseau mulicouche lamellaire à $\lambda=1,33 \mathrm{~nm}$ (caractéristiquesdans le texte)

Experimental and theoretical efficiencies versus the glancing angle of a lamellar multilayer grating for the orders $-1(\mathrm{a}), 0(\mathrm{~b}),+1$ (c) at $\lambda=1.33 \mathrm{~nm}$ ( parameters in the text). 
diffracter un rayonnement de longueur d'onde $\lambda$, incident perpendiculairement aux traits, lorsque les deux conditions suivantes sont vérifiées

$$
\begin{aligned}
& \sin \theta+\sin \theta_{0}=m \frac{\lambda}{d} \\
& \cos \theta-\cos \theta_{0}=p \frac{\lambda}{D}
\end{aligned}
$$

Ces deux relations sont les conditions scalaires de Laue rencontrées en radiocristallographie. Comme pour les MIMs, il convient de leur appliquer une correction d'indice. En combinant les équations (2) et (3), on en déduit une "loi de Bragg généralisée" qui donne l'angle d'attaque $\theta_{0}$ nécessaire à la diffraction à l'ordre $\mathrm{m}$ par la structure multicouche et à l'ordre $\mathrm{p}$ par le réseau latéral:

$$
\mathrm{m} \lambda=\mathrm{d} \sin \theta_{0}\left[1+\sqrt{1-2 \frac{\cos \theta_{0}}{\sin ^{2} \theta_{0}} \frac{\mathrm{p} \lambda}{\mathrm{D}}-\frac{1}{\sin ^{2} \theta_{0}}\left(\frac{\mathrm{p} \lambda}{\mathrm{D}}\right)^{2}}\right]
$$

Cette relation signifie que l'angle d'attaque qui optimise en terme d'efficacité, la diffraction à l'ordre $p$ dépend de la valeur de $p$. La figure 2 illustre ce phénomène [4]. Elle représente la variation expérimentale et théorique en fonction de l'angle d'attaque $\theta_{0}$ de l'efficacité de diffraction d'un rayonnement de longueur d'onde $\lambda=1,33 \mathrm{~nm}$, pour les ordres: $p=-1, p=0$, $\mathrm{p}=+1$. Le réseau comporte 80 bicouches $\mathrm{Mo} / \mathrm{C}$ de période $\mathrm{d}=4,4 \mathrm{~nm}$, la période $\mathrm{D}$ est égale à 1 $\mu \mathrm{m}$ et le rapport cyclique $\Gamma$ vaut 0,5 . On constate que conformément à la relation (4), un ordre $p$ possède un angle d'attaque propre qui maximise son efficacité. Cette dépendance spécifique de l'efficacité de diffraction vis à vis de la longueur d'onde, de l'angle d'attaque et de l'ordre de diffraction du réseau $\mathrm{p}$, sera utilisée pour concevoir un "polychromateur". A incidence quasinormale, ces réseaux pourraient être utilisés comme réseaux dispersifs pour la spectroscopie.

\subsection{Polychromateur}

Si l'on considère un faisceau de rayonnement polychromatique incident à un angle d'attaque donné $\theta_{0}$ sur un réseau lamellaire multicouche, on conçoit d'après ce qui précède que, à la suite de la diffraction, le faisceau va être séparé en plusieurs faisceaux quasi monochromatiques. En effet pour un angle d'attaque fixé, la relation (4) peut être satisfaite pour plusieurs couples de valeurs $(\lambda, p)$. Chaque faisceau qui émerge à un angle $\theta_{p}$ correspond à un ordre p et à une longueur d'onde $\lambda_{\mathrm{p}}$ donnée par la relation:

$$
\lambda_{\mathrm{p}}=2 \frac{\mathrm{m} / \mathrm{d} \sin \theta_{0}-\mathrm{p} / \mathrm{D} \cos \theta_{0}}{\left(\frac{\mathrm{m}}{\mathrm{d}}\right)^{2}+\left(\frac{\mathrm{p}}{\mathrm{D}}\right)^{2}}
$$

L'angle $\theta_{p}$ est relié à l'angle d'attaque $\theta_{0}$, à la longueur d'onde $\lambda_{p}$ et à l'ordre p par la relation (3). Ce comportement d'un réseau lamellaire multicouche sous irradiation polychromatique a été qualifié de "polychromateur". Celui-ci peut être considéré comme un système qui échantillonne la distribution spectrale du rayonnement incident à un intervalle de longueur d'onde $\delta \lambda$ approximativement donné par : 


$$
\delta \lambda=\frac{2 \mathrm{~d}^{2} \cos \theta_{0}}{\mathrm{~m}^{2} \mathrm{D}}
$$

Pour un angle d'attaque fixé, un réseau multicouche et un ordre de réflexion $\mathrm{m}$ donné, cet intervalle est constant . La figure 3 donne un exemple d'un tel comportement [5]. Elle montre la distribution de l'intensité diffractée par le polychromateur en fonction de l'angle d'observation $\theta$. Le polychromateur est irradié par le rayonnement émis par un tube à rayons X à anode de cuivre excité sous une tension de $3 \mathrm{kV}$. Ce rayonnement est́ constitué du rayonnement continu de freinage et de raies d'émission caractéristiques, en particulier la raie $L \alpha(1.33 \mathrm{~nm}, 930 \mathrm{eV})$ et la bande $\mathrm{L} \beta(1.306 \mathrm{~nm}, 950 \mathrm{eV})$ du cuivre. L'angle d'attaque $\theta_{0}$ est fixé à 11,5 degrés. Le polychromateur est un réseau lamellaire gravé dans un miroir multicouche constitué de 115 bicouches $W / B_{4} C$ d'épaisseur $\mathrm{d}=3,1 \mathrm{~nm}$ avec une période $\mathrm{D}=1,33 \mu \mathrm{m}$ et un rapport $\Gamma$ d'environ 0,1 . La valeur de l'ordre de diffraction $p$ est indiquée pour chaque pic. Les énergies du rayonnement associées à chaque ordre $p$ sont portées dans un encart de la figure. L'ordre 0 n'est pas spectralement sélectif et le pic associé contient donc toutes les énergies des rayonnements réfléchis spéculairement. Les pics notés $\alpha$ et $\beta$ correspondent à des énergies incluses dans la largeur spectrale des émissions caractéristiques $L \alpha, \beta$ du cuivre. Ce dispositif polychromateur pourrait se réveler intéressant comme séparateur de faisceau et comme échantillonneur spectral pour source impulsionnelle.

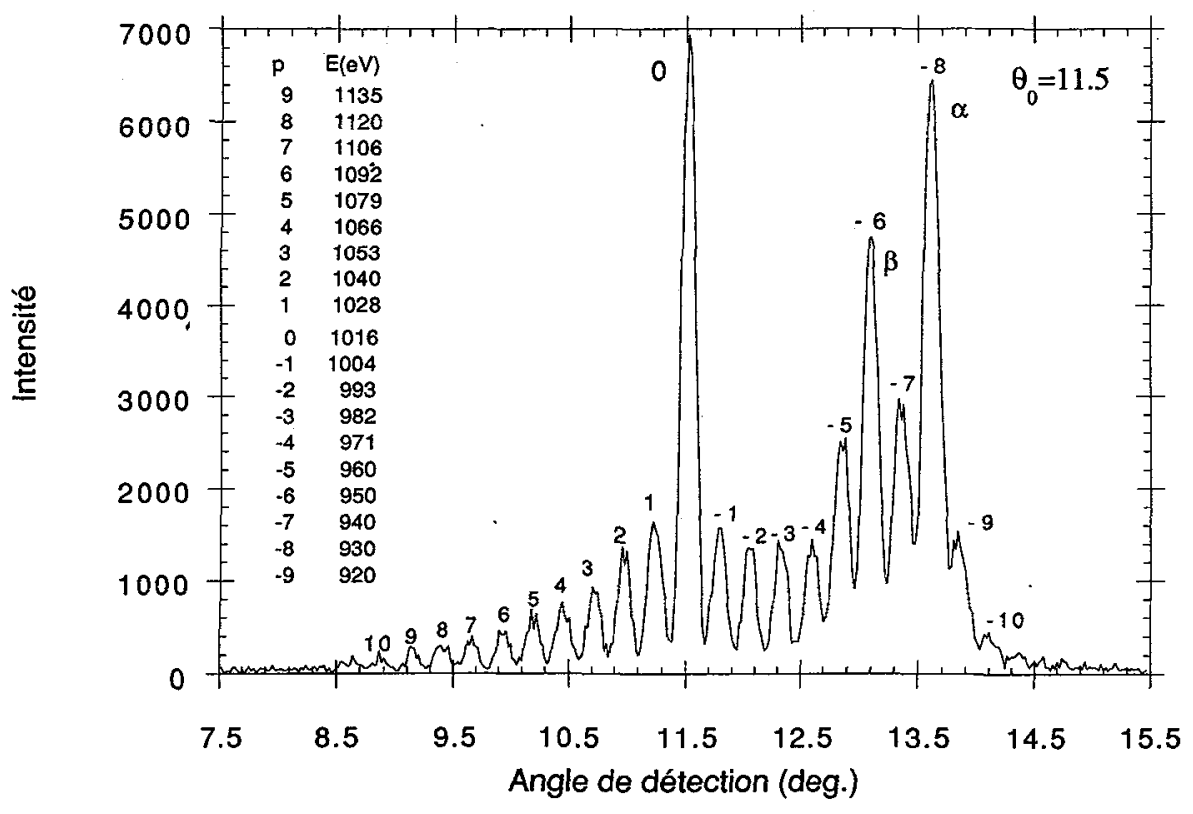

Figure 3: Intensité en fonction de l'angle de détection $\theta$ du rayonnement émis par un tube à rayons $\mathrm{X}$ à anode de cuivre, diffracté par un réseau multicouche lamellaire fonctionnant en mode polychromateur (caractéristiques dans le texte). Dans l'encart sont indiquées les énergies du rayonnement associées à chaque ordre $p$.

Intensity versus the detection angle $\theta$ of the radiation emitted by an $x$-ray tube with a copper anode and diffracted by a lamellar multilayer grating operating as a polychromator (parameters in the text). In the inset the radiation energies associated with each order $p$ are given. 


\subsection{Monochromateur multicouche à bande passante étroite}

Les miroirs interférentiels multicouches utilisés usuellement pour l'analyse spectrale présentent une bande passante relativement large donnant lieu à une résolution spectrale médiocre $\left(\Delta \lambda / \lambda \approx 10^{-2}\right)$. Cette valeur empêche l'utilisation des MIMs pour la spectroscopie X-UV haute résolution telle que l'on peut la pratiquer dans le domaine du rayonnement $X$ dur avec des cristaux naturels. On peut comprendre l'origine de ces larges bandes passantes en considérant les MIMs comme des filtres interférentiels. Pour obtenir une résolution importante il est nécessaire que le nombre d'éléments diffractants agissant dans ce filtre soit suffisamment grand. Dans le cas des MIMs utilisés dans le domaine X-UV les éléments diffractants sont les bicouches de l'empilement. Cependant même si l'on dispose d'un grand nombre de bicouches, le nombre de ces dernières contribuant efficacement à la diffraction sera limité par la "dépopulation de l'onde incidente" par suite de la photoabsorption et de la réflexion spéculaire à chaque interface. Une technique pour diminuer la largeur de la bande passante des MIMs, donc pour améliorer la résolution, consiste à faciliter la pénétration de l'onde incidente dans la profondeur de la structure. Ceci peut être réalisé par la gravure qui crée dans la structure des zones de vide (les traits) et provoque donc "l'allègement" du milieu ce qui facilite la pénétration de l'onde incidente. Le coefficient d'absorption moyen du milieu ainsi que le coefficient de réflexion aux interfaces sont pratiquement multipliés par le facteur $\Gamma$-par rapport à leur valeur dans le MIM-. La conception d'un monochromateur multicouche à bande passante étroite performant consiste à déterminer les paramètres (nombre de bicouches, rapport cyclique $\Gamma$, nature des matériaux) pour obtenir une bande passante de faible largeur, tout en conservant une efficacité pic élevée ( de l'ordre de $80 \%$ de la réflectivité pic du miroir non gravé correspondant). Ceci nécessite le calcul des performances d'un réseau multicouche au moyen d'une théorie "dynamique", qui prend en compte globalement la photoabsorption et la diffusion multiple [6]. Notons que pour un MIM la largeur de la bande passante tend asymptotiquement vers une valeur non nulle tandis que pour un réseau gravé, elle tend vers une valeur nulle quand le nombre de bicouches tend vers l'infini.

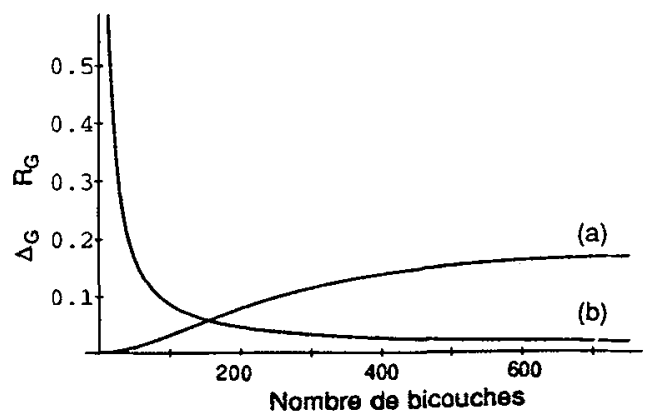

Figure 4: Variations théoriques à $\lambda=1,33 \mathrm{~nm}$ en fonction du nombre de bicouches, de l'efficacité pic à l'ordre 0 ( $R_{G}$, courbe b) et de la largeur de la bande passante $\left(\Delta_{G}\right.$ en degré, courbe a) d'un réseau lamellaire gravé dans un MIM avec $\Gamma=0.05$ (caractéristiques dans le texte).

Theoretical peak efficiency at the 0 order $\left(R_{G}\right.$, curve $b$ ) and bandwidth ( $\Delta_{G}$ in degree. curve a) versus the bilayer number of a lamellar grating patterned in a multilayer interferential mirror (MIM) with $\Gamma=0.05$ (parameters in the text). 
En pratique on réalise un réseau lamellaire du type de celui étudié précédemment, avec une faible valeur du rapport $\Gamma$ (typiquement $0,05-0,15$ ) et un nombre élevé de bicouches (environ 200 à $1 \mathrm{keV})$ que l'on utilise à l'ordre zéro $(\mathrm{p}=0)$. La théorie dynamique de la diffraction des réseaux appliquée dans le domaine X-UV a confirmé la faisabilité de principe d'un tel monochromateur. Ainsi la figure 4 [6] illustre les performances que l'on peut attendre avec ce type de monochromateur à la longueur d'onde $\lambda=1,33 \mathrm{~nm}$ à partir d'un MIM constitué de bicouches Mo/C d'épaisseur $\mathrm{d}=3 \mathrm{~nm}$; la période $\mathrm{D}$ vaut $1 \mu \mathrm{m}$ et le rapport cyclique $\Gamma=0,05$. En pratique la réalisation d'un empilement régulier possédant un grand nombre de bicouches reste encore délicate; il se pose en particulier des problèmes de dérive dans les conditions de dépôt lorsque le temps de fabrication s'accroit. Néanmoins la réduction de la bande passante par gravure a été vérifiée expérimentalement [4],[7]. La figure 5 [4] présente le spectre du rayonnement émis par un tube à rayons $\mathrm{X}$ à anode d'aluminium obtenu dans la région $\mathrm{Al} \mathrm{K} \alpha$ avec un MIM (150 bicouches de W/Si, $d=2,4 \mathrm{~nm}$ ) et avec le monochromateur gravé dans ce MIM avec $D=1 \mu \mathrm{m}$ et $\Gamma=0,16$.
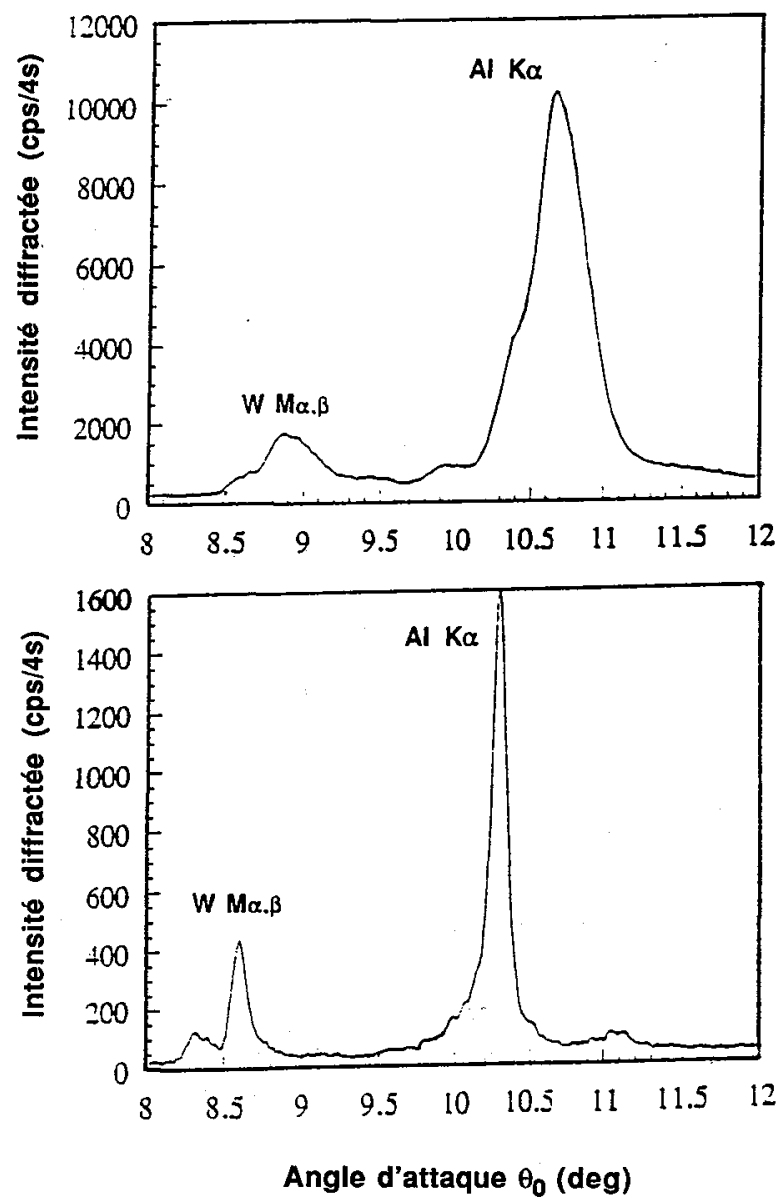

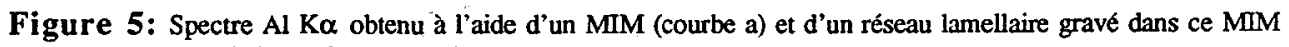
(courbe b) (caractériutiques dans le texte) (parameters in the text). 
La gravure en diminuant la densité moyenne de la structure provoque aussi une diminution de la valeur de l'angle d'attaque critique $\theta c$ par rapport au miroir non gravé. La zone dé réflexion quasi totale est ainsi réduite. Ce phénomène est illustré par la figure 6 qui montre les variations expérimentales en fonction de l'angle d'attaque $\theta_{0}$ de la réflectivité spéculaire d'un MIM et du réseau lamellaire gravé dans ce $\mathrm{MIM}$ avec $\mathrm{D}=1,33 \mu \mathrm{m}$ et $\Gamma=0,1$. La longueur d'onde est celle de l'émission $\mathrm{K}$ du carbone $(\lambda=4,4 \mathrm{~nm})$. Le MIM est constitué de 115 bicouches $\mathrm{W} / \mathrm{B}_{4} \mathrm{C}$ d'épaisseur $\mathrm{d}=3,1 \mathrm{~nm}$.

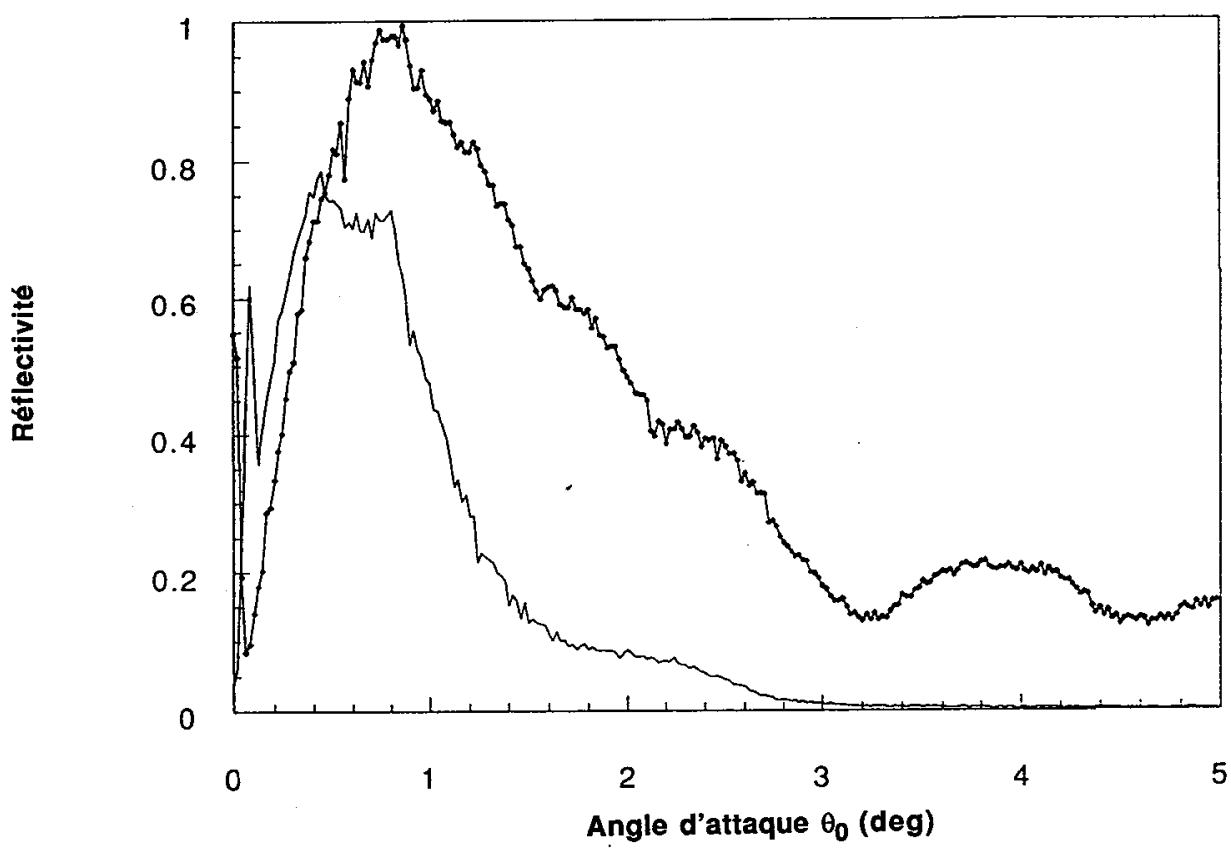

Figure 6: Variation expérimentale de réflectivité spéculaire en fonction de l'angle d'attaque à $\lambda=4,4 \mathrm{~nm}$ d'un MIM (trait épais) et d'un réseau gravé dans ce MIM (trait fin) avec un rapport $\Gamma=0,1$ (caractéristiques dans le texte)

Experimental specular reflectivity versus the glancing angle at $\lambda=4.4 \mathrm{~nm}$ of a multilayer interferential mirror- MIM- (thick line) and a lamellar grating (thin line) etched in this MIM with $\Gamma=0.1$ (parameters in the text).

Cet effet exploité à plus grande longueur d'onde pour les monochromateurs équipant les spectromètres à rayons $\mathrm{X}$ mous devrait permettre d'améliorer les performances de ceux-ci en terme de réduction du rapport signal utile/fond [8]. En effet l'intensité réfléchie spéculairement au delà de $\theta \mathrm{c}$ est fortement réduite par la gravure pour des faibles valeurs de $\Gamma$ et devrait moins perturber l'observation d'un pic de Bragg situé dans ce domaine. Ceci est illustré par la figure 7. Celle-ci représente la réflectivité d'un MIM constitué de 200 bicouches $\mathrm{Mo} / \mathrm{B}_{4} \mathrm{C}$ d'épaisseur $\mathrm{d}=6,5 \mathrm{~nm}$, comparativement à l'efficacité à l'ordre zéro d'un réseau lamellaire gravé dans ce MIM avec $\Gamma=0,25$. Il s'agit de valeurs calculées pour la longueur d'onde de l'émission $K$ du béryllium, $\lambda=11,3 \mathrm{~nm}$. On constate la forte diminution de l'importance de la réflexion spéculaire non sélective. 


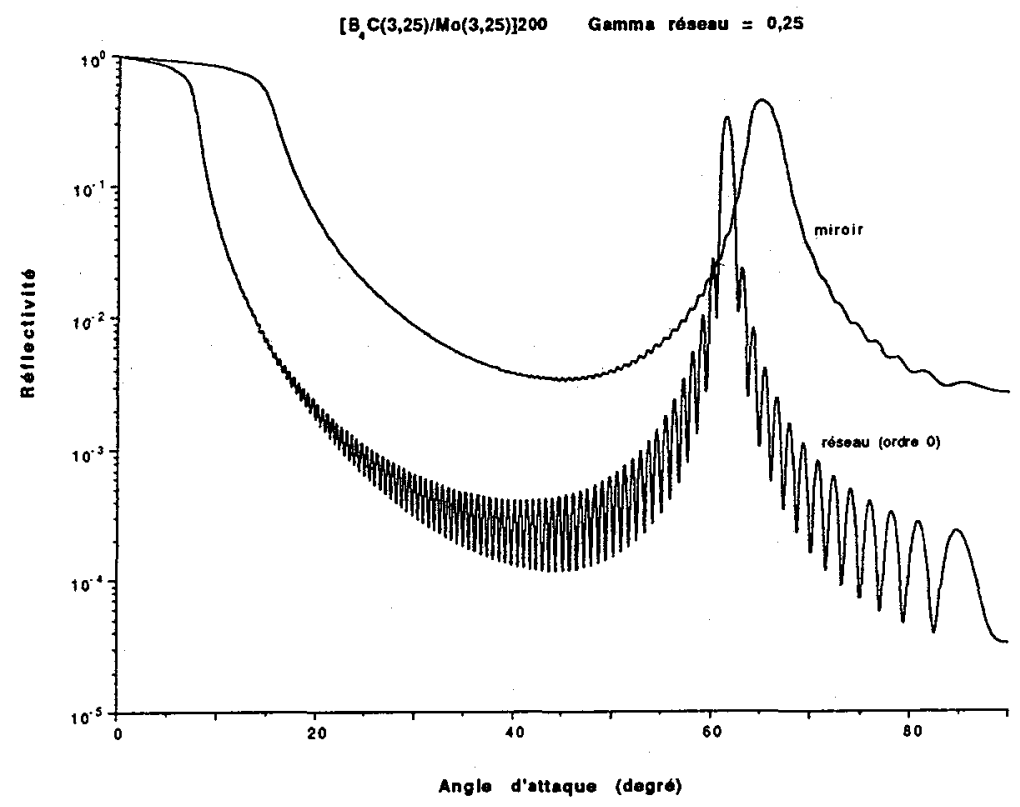

Figure 7: Réflectivité d'un MIM et efficacité à l'ordre zéro d'un réseau multicouche gravé dans ce MIM avec $\Gamma=0,25$ à la longueur d'onde $\lambda=11,3 \mathrm{~nm}$ (caractéristiques dans le texte).

Reflectivity of a MIM and zero order efficiency of a lamellar grating pattemed in this MIM with $\Gamma=$ 0.25 at the wavelength $\lambda=11.3 \mathrm{~nm}$ (parameters in the text).

\section{STRUCTURES APERIODIQUES: LENTILLES DE BRAGG-FRESNEL}

Les techniques actuelles de microfabrication permettent aussi l'écriture de motifs non périodiques. Cette possibilité a été exploitée pour réaliser des lentilles dites de Bragg-Fresnel. L'idée est de combiner le principe des lentilles de Fresnel et la réflexion de Bragg. Dans une lentille de Fresnel on fait interagir une onde incidente avec une "surface optique" structurée de telle sorte que les ondes sortantes, par un phénomène d'interférences constructives occasionné par le motif sur la surface, soient des ondes quasi sphériques convergeant vers une série de foyers. Initialement cette méthode a été appliquée dans le mode "transmission", c'est à dire que la surface est un plan, la source étant située dans l'un des demi-espaces limité par ce plan, les foyers (réels) étant dans l'autre demi-espace. Dans le cas d'une symétrie radiale les motifs dessinés sur la surface sont des cercles concentriques de rayon croissant selon la racine carré des nombres entiers et formant des couronnes alternativement transparentes et opaques (zones de Soret). Cependant le principe est aussi applicable par réflexion, source et foyers étant situés dans le même demi-espace. Dans ce cas en symétrie radiale les motifs deviennent des ellipses. La. réflexion peut être assurée par la diffraction dans les conditions de Bragg. Le principe et des études plus approfondies des ces lentilles de Bragg-Fresnel ont été publiés [9-12]. Des lentilles "elliptiques" multicouches permettent une focalisation à deux dimension ont été récemment réalisées et équipent une microsonde par fluorescence travaillant dans le domaine 8-12 keV $[13,14]$. 


\section{CONCLUSIONS}

La combinaison des techniques de dépôt des films d'épaisseur nanométrique et des techniques de microfabrication issues de la microélectronique permet pour l'optique X-UV, le développement de nouveaux composants basés sur des structures multicouches gravées. Ceux-ci devraient offrir dans un proche avenir des outils performants pour l'analyse spectrale (réseaux de diffraction, monochromateurs à bande passante étroite, polychromateurs), pour la microanalyse ainsi que l'imagerie (lentilles de Fresnel). Des applications plus spécifiques: coupleurs de sortie pour laser $\mathrm{X}$, composants pour ligne de lumière dans les synchrotrons ou dispositifs de diagnostic, sont à l'étude. A plus long terme, des applications en interférométrie et holographie sont envisageables en coïncidence avec le développement de sources X-UV cohérentes (synchrotron de troisième génération, laser).

\section{Références}

[1] Khan Malek C., J. of X-Ray Sc. Techno. 3 (1991)45-67

[2] Pardo B. et al, Revue Phys. Appl. 23 (1988)1579-1597.

[3] Dhez P., Ann. Phys. Fr.. 15 (1990) 493-527.

[4] André J-M. et al., J.Phys. III France 4 (1994)1659-1668.

[5] Krastev K. et al., accepté au Nucl. Instrum. Methods A (Juin 1995).

[6] Sammar A. et al., C. R. Acad. Sci. Paris, t.316, Série II (1993)1055-1060.

[7] Bac S. et al., J. of X-Ray Sc.Techno. 5 (1995)161-180.

[8] Fialin M.et al., soumis à J. of X-ray Spectro. (1995).

[9] Aristov V. V.et al., Rev. Phys. Appl. 23 (1988)1623-1630.

[10] Erko A. I., J. of X-Ray Sc. Techno. 2 (1990)297-316.

[11] André J-M., Barchewitz R., Ann. Phys. Paris 19 (1994)C1-17.

[12] Idir M. et al., "Characterisation of linear Bragg-Fresnel multilayer lenses at $7 \AA$ and comparison with theoretical calculations", 4th International Conference on X-ray Microscopy, Chernogoloska, Russie, 20-24 Septembre 1993.

[13] Erko A.I. et al., Opt. Comm . 106 (1994)146-149.

[14] Chevallier P.et al, Nucl. Instrum. Methods A A 354 (1995)584-587. 